\title{
DOUBT FUZZY BCI-ALGEBRAS
}

\section{ZHAN JIANMING and TAN ZHISONG}

Received 10 August 2001

\begin{abstract}
The aim of this note is to introduce the notion of doubt fuzzy $p$-ideals in BCI-algebras and to study their properties. We also solve the problem of classifying doubt fuzzy $p$-ideals and study fuzzy relations on BCI-algebras.
\end{abstract}

2000 Mathematics Subject Classification: 03G25, 06F35, 03E72.

1. Introduction and preliminaries. The concept of a fuzzy set is applied to generalize some of the basic concepts of general topology [2]. Rosenfeld [6] constituted a similar application to the elementary theory of groupoids and groups. Xi [7] applied the concept of fuzzy set to BCK-algebras. Jun [4] defined a doubt fuzzy subalgebra, doubt fuzzy ideal, doubt fuzzy implicative ideal, and doubt fuzzy prime ideal in BCIalgebras, and got some results about it. In this note, we define a doubt fuzzy $p$-ideal of a BCI-algebra and investigate its properties.

A mapping $f: X \rightarrow Y$ of BCI-algebras is called homomorphism if $f(x * y)=f(x) *$ $f(y)$ for all $x, y \in X$. A nonempty subset $I$ of a BCI-algebra $X$ is called an ideal of $X$ if (i) $0 \in I$, (ii) $x * y \in I$ and $y \in I$ imply that $x \in I$. We recall that a fuzzy subset $\mu$ of a set $X$ is a function $\mu$ from $X$ into [0,1]. Let $\operatorname{Im} \mu$ denote the image set of $\mu$. We will write $a \wedge b$ for $\min \{a, b\}$, and $a \vee b$ for $\max \{a, b\}$, where $a$ and $b$ are any real numbers.

Given a fuzzy set $\mu$ and $t \in[0,1]$, let $\mu_{t}=\{x \in X \mid \mu(x) \geq t\}$ and $\mu^{t}=\{x \in X \mid$ $\mu(x) \leq t\}$. These could be empty sets. The set $\mu_{t} \neq \varnothing$ (resp., $\mu^{t} \neq \varnothing$ ) is called the $t$-confidence (resp., $t$-doubt) set of $\mu$ (see [3]).

DEFINITION 1.1 (see [7]). For any $x, y$ in a BCI-algebra $X$,

(i) if $\mu(x * y) \geq \mu(x) \wedge \mu(y)$, then $\mu$ is called a fuzzy subalgebra of $X$;

(ii) if $\mu(0) \geq \mu(x)$ and $\mu(x) \geq \mu(x * y) \wedge \mu(y)$, then $\mu$ is called a fuzzy ideal of $X$.

Definition 1.2 (see [4]). Let $X$ be a BCI-algebra. A fuzzy set $\mu$ in $X$ is called (i) a doubt fuzzy subalgebra (briefly, DF-subalgebra) of $X$ if $\mu(x * y) \leq \mu(x) \vee \mu(y)$ for all $x, y \in X$; and (ii) a doubt fuzzy ideal (briefly, DF-ideal) of $X$ if $\mu(0) \leq \mu(x)$ and $\mu(x) \leq \mu(x * y) \vee \mu(y)$ for all $x, y \in X$.

DefinItion 1.3 (see [5]). A nonempty subset $I$ of BCI-algebra $X$ is called $p$-ideal if

(i) $0 \in I$;

(ii) $(x * z) *(y * z) \in I$ and $y \in I$ imply that $x \in I$ for all $x, y, z \in X$.

Definition 1.4 (see [5]). A fuzzy subset $\mu$ of a BCI-algebra $X$ is called a fuzzy $p$-ideal of $X$ if

(i) $\mu(0) \geq \mu(x)$ for any $x \in X$;

(ii) $\mu(x) \geq \mu((x * z) *(y * z)) \wedge \mu(y)$ for any $x, y, z \in X$. 


\section{Doubt fuzzy $p$-ideals}

DEFINITION 2.1. A fuzzy subset $\mu$ of a BCI-algebra $X$ is called a doubt fuzzy $p$-ideal (briefly, DF $p$-ideal) of $X$ if

(i) $\mu(0) \leq \mu(x)$ for any $x \in X$;

(ii) $\mu(x) \leq \mu((x * z) *(y * z)) \vee \mu(y)$ for any $x, y, z \in X$.

EXAMPLE 2.2. Let $X=\{0, a, b, c\}$ in which $*$ is defined by

\begin{tabular}{c|cccc}
$*$ & 0 & $a$ & $b$ & $c$ \\
\hline 0 & 0 & $a$ & $b$ & $c$ \\
$a$ & $a$ & 0 & $c$ & $b$ \\
$b$ & $b$ & $c$ & 0 & $a$ \\
$c$ & $c$ & $c$ & $b$ & 0
\end{tabular}

Then $(X ; *, 0)$ is a BCI-algebra. Let $t_{0}, t_{1}, t_{2} \in[0,1]$ be such that $t_{0}<t_{1}<t_{2}$. Define $\mu: X \rightarrow[0,1]$ by $\mu(0)=t_{0}, \mu(a)=t_{1}$, and $\mu(b)=\mu(c)=t_{2}$. Routine calculations give that $\mu$ is a DF $p$-ideal of $X$.

Proposition 2.3. If $\mu$ is a DF $p$-ideal of a BCI-algebra $X$, then $\mu(x) \leq \mu(0 *(0 * x))$ for all $x \in X$.

Proof. Since $\mu$ is a DF $p$-ideal of $X$, we have $\mu(x) \leq \mu((x * x) *(0 * x)) \vee \mu(0)=$ $\mu(0 *(0 * x)) \vee \mu(0)=\mu(0 *(0 * x))$.

Proposition 2.4. Every DF $p$-ideal is a DF-ideal.

Proof. Let $\mu$ be a DF $p$-ideal of $X$. We have $\mu(x) \geq \mu((x * 0) *(y * 0)) \vee \mu(y)=$ $\mu(x * y) \vee \mu(y)$ for all $x, y \in X$. Hence $\mu$ is a DF-ideal.

REMARK 2.5. The converse of Proposition 2.4 is not true in general as shown in the following example.

EXAMPLE 2.6. Let $X=\{0, a, 1,2,3\}$ in which $*$ is defined by

\begin{tabular}{l|lllll}
$*$ & 0 & $a$ & 1 & 2 & 3 \\
\hline 0 & 0 & 0 & 3 & 2 & 1 \\
$a$ & $a$ & 0 & 3 & 2 & 1 \\
1 & 1 & 1 & 0 & 3 & 2 \\
2 & 2 & 2 & 1 & 0 & 3 \\
3 & 3 & 3 & 2 & 1 & 0
\end{tabular}

Then $X$ is a BCI-algebra. Let $t_{0}, t_{1}, t_{2} \in[0,1]$ be such that $t_{0}<t_{1}<t_{2}$. Define $\mu$ : $X \rightarrow[0,1]$ by $\mu(0)=t_{0}, \mu(a)=t_{1}$, and $\mu(1)=\mu(2)=\mu(3)=t_{3}$. Routine calculations give that $\mu$ is a DF-ideal of $X$. But $\mu$ is not a DF $p$-ideal of $X$, because $\mu(a)=t_{1}$, and $\mu((a * 1) *(0 * 1)) \vee \mu(0)=\mu(0)=t_{0}$, that is, $\mu(a)>\mu((a * 1) *(0 * 1)) \vee \mu(0)$.

Proposition 2.7. If $\mu$ is a DF $p$-ideal of a BCI-algebra $X$, then $\mu(x * y) \geq \mu((x *$ $z) *(y * z))$ for all $x, y, z \in X$.

Proof. Note that in a BCI-algebra $X$ the inequality $(x * z) *(y * z) \leq x * y$ holds. It follows that $((x * z) *(y * z)) *(x * y)=0$. Since $\mu$ is a DF-ideal by Proposition 2.4. 
We have $\mu((x * z) *(y * z)) \geq \mu(((x * z) *(y * z)) *(x * y)) \vee \mu(x * y)=\mu(0) \vee$ $\mu(x * y)=\mu(x * y)$. This completes the proof.

Proposition 2.8. Let $\mu$ be a DF-ideal of a BCI-algebra X. If $\mu$ satisfies $\mu(x * y) \leq$ $\mu((x * z) *(y * z))$ for any $x, y, z \in X$, then $\mu$ is a DF $p$-ideal of $X$.

Proof. Let $\mu$ be a DF-ideal of $X$ satisfying $\mu(x * y) \leq \mu((x * z) *(y * z))$ for all $x, y, z \in X$. Then $\mu((x * z) *(y * z)) \vee \mu(y) \geq \mu(x)$. This completes the proof.

Proposition 2.9. Let $\mu$ be a DF-ideal of a BCI-algebra X. Then $\mu(0 *(0 * x)) \leq \mu(x)$ for all $x \in X$.

Proof. We have that $\mu(0 *(0 * x)) \leq \mu((0 *(0 * x)) * x) \vee \mu(x)=\mu(0) \vee \mu(x)=$ $\mu(x)$ for all $x \in X$.

Proposition 2.10. Let $\mu$ be a DF-ideal of a BCI-algebra $X$ satisfying $\mu(0 *(0 * x)) \geq$ $\mu(x)$ for all $x \in X$.

Proof. Let $x, y, z \in X$. Then

$$
\begin{aligned}
\mu((x * z) *(y * z)) & \geq \mu(0 *(0 *((x * z) *(y * z)))) \\
& =\mu((0 * y) *(0 * x)) \\
& =\mu(0 *(0 *(x * y))) \\
& \geq \mu(x * y)
\end{aligned}
$$

It follows from Proposition 2.8 that $\mu$ is a DF $p$-ideal of $X$.

THEOREM 2.11. Let $\mu$ be a fuzzy subset of a BCI-algebra X. If $\mu$ is a DF $p$-ideal of $X$, then the set $I=\{x \in X \mid \mu(x)=\mu(0)\}$ is a $p$-ideal of $X$.

Proof. Assume that $\mu$ is a DF $p$-ideal of $X$. Clearly $0 \in I$. Let $(x * z) *(y * z) \in I$ and $y \in I$. Then $\mu(x) \leq \mu((x * z) *(y * z)) \vee \mu(y)=\mu(0)$. But $\mu(0) \leq \mu(x)$ for all $x \in X$. Thus $\mu(0)=\mu(x)$. Hence $x \in I$. This completes the proof.

Definition 2.12 (see [6]). Let $f$ be a mapping defined on a set $X$. If $\mu$ is a fuzzy subset of $X$, then the fuzzy subset $v$ of $f(x)$, defined by

$$
v(y)=\inf _{x \in f^{-1}(y)} \mu(x)
$$

for all $y \in f(x)$, is called the image of $\mu$ under $f$. Similarly, if $v$ is a fuzzy subset of $f(x)$, then the fuzzy subset $\mu=v \circ f$ in $X$ (i.e., the fuzzy subset defined by $\mu(x)=$ $v(f(x))$ for all $x \in X)$ is called the preimage of $v$ under $f$.

THEOREM 2.13. An onto homomorphic preimage of a DF p-ideal is also a DF $p$ ideal.

Proof. Let $f: X \rightarrow X^{\prime}$ be an onto homomorphism of BCI-algebras, $v$ a DF $p$-ideal of $X^{\prime}$, and $\mu$ the preimage of $v$ under $f$. Then $v(f(x))=\mu(x)$ for all $x \in X$. Since $f(x) \in X^{\prime}$ and $v$ is a DF $p$-ideal of $X^{\prime}$, it follows that $v\left(0^{\prime}\right) \leq v(f(x))=\mu(x)$ for all $x \in X$, where $0^{\prime}$ is the zero element of $X^{\prime}$. But $v\left(0^{\prime}\right)=v(f(0)=\mu(0))$, and so $\mu(0) \leq \mu(x)$ for all $x \in X$. 
Since $v$ is a DF $p$-ideal, we have $\mu(x)=v(f(x)) \leq v\left(\left(f(x) * z^{\prime}\right) *\left(y^{\prime} * z^{\prime}\right)\right) \vee v\left(y^{\prime}\right)$ for any $y^{\prime}, z^{\prime} \in X^{\prime}$. Since $f$ is onto, there exist $y, z \in X$ such that $f(y)=y^{\prime}$ and $f(z)=z^{\prime}$. Then

$$
\begin{aligned}
\mu(x) & \leq v\left(\left(f(x) * z^{\prime}\right) *\left(y^{\prime} * z^{\prime}\right)\right) \vee v\left(y^{\prime}\right) \\
& =v((f(x) * f(z)) *(f(y) * f(z))) \\
& =v(f(x * z) * f(y * z)) \vee v(f(y)) \\
& =v(f(x * z) *(y * z)) \vee v(f(y)) \\
& =\mu((x * z) *(y * z)) \vee \mu(y) .
\end{aligned}
$$

Since $y^{\prime}$ and $z^{\prime}$ are arbitrary elements of $X^{\prime}$, the above result is true for all $y, z \in X$, that is, $\mu(x) \leq \mu((x * z) *(y * z)) \vee \mu(y)$ for all $x, y, z \in X$. This completes the proof.

Definition 2.14 (see [6]). A fuzzy subset $\mu$ of $X$ has inf property if for any subset $T$ of $X$, there exists $t_{0} \in T$ such that

$$
\mu\left(t_{0}\right)=\inf _{t \in T} \mu(t)
$$

THEOREM 2.15. An onto homomorphic image of a DF $p$-ideal with inf property is a DF $p$-ideal.

Proof. Let $f: X \rightarrow X^{\prime}$ be an onto homomorphism of BCI-algebras, $\mu$ a DF $p$-ideal of $X$ with inf property, and $v$ the image of $\mu$ under $f$. Since $\mu$ is a DF $p$-ideal of $X$, we have $\mu(0) \leq \mu(x)$ for all $x \in X$. Note that $0 \in f^{-1}\left(0^{\prime}\right)$, where 0 and $0^{\prime}$ are the zero elements of $X$ and $X^{\prime}$, respectively. Thus $v\left(0^{\prime}\right)=\inf _{t \in f^{-1}\left(0^{\prime}\right)} \mu(t)=\mu(0) \leq \mu(x)$ for all $x \in X$, which implies that $v\left(0^{\prime}\right) \leq \inf _{t \in f^{-1}\left(x^{\prime}\right)} \mu(t)=v\left(x^{\prime}\right)$ for any $x^{\prime} \in X^{\prime}$. For any $x^{\prime}, y^{\prime}, z^{\prime} \in X^{\prime}$, let $x_{0} \in f^{-1}\left(x^{\prime}\right), y_{0} \in f^{-1}\left(y^{\prime}\right)$, and $z_{0} \in f^{-1}\left(z^{\prime}\right)$ be such that

$$
\begin{gathered}
\mu\left(x_{0}\right)=\inf _{t \in f^{-1}\left(x^{\prime}\right)} \mu(t), \quad \mu\left(y_{0}\right)=\inf _{t \in f^{-1}\left(y^{\prime}\right)} \mu(t), \\
\mu\left(\left(x_{0} * z_{0}\right) *\left(y_{0} * z_{0}\right)\right)=\inf _{t \in f^{-1}\left(\left(x^{\prime} * z^{\prime}\right) *\left(y^{\prime} * z^{\prime}\right)\right)} \mu(t) .
\end{gathered}
$$

Then

$$
\begin{aligned}
v\left(x^{\prime}\right) & =\inf _{t \in f-1\left(x^{\prime}\right)} \mu(t) \\
& =\mu\left(x_{0}\right) \leq \mu\left(\left(x_{0} * z_{0}\right) *\left(y_{0} * z_{0}\right)\right) \vee \mu\left(y_{0}\right) \\
& =\inf _{t \in f^{-1}\left(\left(x^{\prime} * z^{\prime}\right) *\left(y^{\prime} * z^{\prime}\right)\right)} \mu(t) \vee \inf _{t \in f^{-1}\left(y^{\prime}\right)} \mu(t) \\
& =v\left(\left(x^{\prime} * z^{\prime}\right) *\left(y^{\prime} * z^{\prime}\right)\right) \vee v\left(y^{\prime}\right) .
\end{aligned}
$$

Hence $v$ is a DF $p$-ideal of $X^{\prime}$.

THEOREM 2.16. A fuzzy subset $\mu$ of a BCI-algebra $X$ is a DF $p$-ideal if and only if, for every $t \in[0,1], \mu^{t}$ is a $p$-ideal of $X$, when $\mu^{t} \neq \varnothing$. 
Proof. Assume that $\mu$ is a DF $p$-ideal of $X$. By Definition 2.1, we have $\mu(0) \leq \mu(x)$ for any $x \in X$. Therefore, $\mu(0) \leq \mu(x) \leq t$ for $x \in \mu^{t}$, and so $0 \in \mu^{t}$. Let $(x * z) *$ $(y * z) \in \mu^{t}$ and $y \in \mu^{t}$. Since $\mu$ is a DF $p$-ideal, it follows that $\mu(x) \leq \mu((x * z) *$ $(y * z)) \vee \mu(y) \leq t$, and that $x \in \mu^{t}$. Hence $\mu^{t}$ is a $p$-ideal of $X$. Conversely, we only need to show that $\mu$ is a DF $p$-ideal of $X$. If Definition 2.1(i) is not true, then there exists $x^{\prime} \in X$ such that $\mu(0)>\mu\left(x^{\prime}\right)$. If we take $t^{\prime}=\left(\mu\left(x^{\prime}\right)+\mu(0)\right) / 2$, then $\mu(0)>t^{\prime}$ and $0 \leq \mu\left(x^{\prime}\right)<t^{\prime} \leq 1$. Thus $x^{\prime} \in \mu^{t^{\prime}}$ and $\mu^{t^{\prime}} \neq \varnothing$. As $\mu^{t^{\prime}}$ is a $p$-ideal of $X$, we have $0 \in \mu^{t^{\prime}}$, and so $\mu(0) \leq t^{\prime}$. This is a contradiction. Now assume that Definition 2.1(ii) is not true. Suppose that there exist $x^{\prime}, y^{\prime}, z^{\prime} \in X$ such that $\mu\left(x^{\prime}\right)>\mu\left(\left(x^{\prime} * z^{\prime}\right) *\left(y^{\prime} *\right.\right.$ $\left.\left.z^{\prime}\right)\right) \vee \mu\left(y^{\prime}\right)$. Putting $t^{\prime}=\left(\mu\left(x^{\prime}\right)+\mu\left(\left(x^{\prime} * z^{\prime}\right) *\left(y^{\prime} * z^{\prime}\right)\right) \vee \mu\left(y^{\prime}\right)\right) / 2$, then $\mu\left(x^{\prime}\right)>t^{\prime}$ and $0 \leq \mu\left(\left(x^{\prime} * z^{\prime}\right) *\left(y^{\prime} * z^{\prime}\right)\right) \vee \mu\left(y^{\prime}\right) \leq 1$. Hence, $\mu\left(\left(x^{\prime} * z^{\prime}\right) *\left(y^{\prime} * z^{\prime}\right)\right)<t^{\prime}$ and $\mu\left(y^{\prime}\right)<t^{\prime}$, which imply that $\left(x^{\prime} * z^{\prime}\right) *\left(y^{\prime} * z^{\prime}\right) \in \mu^{t^{\prime}}$ and $y^{\prime} \in \mu^{t^{\prime}}$, since $\mu^{t^{\prime}}$ is a $p$ ideal, it follows that $x^{\prime} \in \mu^{t^{\prime}}$, and $\mu\left(x^{\prime}\right) \leq t^{\prime}$. This is also a contradiction. Hence, $\mu$ is a DF $p$-ideal of $X$.

COROLlary 2.17. If a fuzzy subset $\mu$ of a BCI-algebra $X$ is a DF $p$-ideal, then for every $t \in \operatorname{Im} \mu$, $\mu^{t}$ is a $p$-ideal of $X$, when $\mu^{t} \neq \varnothing$.

\section{Doubt Cartesian product of doubt fuzzy $p$-ideals}

Definition 3.1 (see [1]). A fuzzy relation on any set $S$ is a fuzzy subset $\mu: S \times S \rightarrow$ $[0,1]$.

Definition 3.2. If $\mu$ is a fuzzy relation on a set $S$ and $v$ is a fuzzy subset of $S$, then $\mu$ is a doubt fuzzy relation on $v$ if $\mu(x, y) \geq \mu(x) \vee \mu(y)$ for all $x, y \in S$.

Definition 3.3. Let $\mu$ and $v$ be fuzzy subsets of a set $S$. The doubt Cartesian product of $\mu$ and $v$ is defined by $(\mu \times v)(x, y)=\mu(x) \vee v(y)$ for all $x, y \in S$.

LEMMA 3.4. Let $\mu$ and $v$ be fuzzy subsets of a set $S$. Then (i) $\mu \times v$ is a fuzzy relation on $S$; (ii) $(\mu \times v)_{t}=\mu_{t} \times v_{t}$ for all $t \in[0,1]$.

DEFinITION 3.5. If $v$ is a fuzzy subset of a set $S$, the smallest doubt fuzzy relation on $S$ that is a doubt fuzzy relation on $v$ is $\mu_{v}$, given by $\mu_{v}(x, y)=v(x) \vee v(y)$ for all $x, y \in S$.

LEMмA 3.6. For a given fuzzy subset $v$ of a set $S$, let $\mu_{v}$ be the smallest doubt fuzzy relation on a set $S$. Then for $t \in[0,1],\left(\mu_{v}\right)_{t}=v_{t} \times v_{t}$.

Proposition 3.7. For a given fuzzy subset $v$ of a BCI-algebra $X$, let $\mu_{v}$ be the smallest doubt fuzzy relation on $X$. If $\mu_{v}$ is a DF $p$-ideal of $X \times X$, then $v(x) \geq v(0)$ for all $x \in X$.

Proof. Since $\mu_{v}$ is a DF $p$-ideal of $X \times X$, it follows that $\mu_{v}(x, x) \geq \mu_{v}(0,0)$, where $(0,0)$ is the zero element of $X \times X$. But this means that $v(x) \vee v(x) \geq v(0) \vee v(0)$, which implies that $v(x) \geq v(0)$.

THEOREM 3.8. Let $\mu$ and $v$ be DF $p$-ideal of a BCI-algebra $X$. Then $\mu \times v$ is a DF p-ideal of $X \times X$. 
Proof. Note first that for every $(x, y) \in X \times X,(\mu \times v)(0,0)=\mu(0) \vee v(0) \leq v(x) \vee$ $v(y)=(\mu \times v)(x, y)$. Now let $\left(x_{1}, x_{2}\right),\left(y_{1}, y_{2}\right),\left(z_{1}, z_{2}\right) \in X \times X$. Then

$$
\begin{aligned}
(\mu \times & v)\left(\left(\left(x_{1}, x_{2}\right) *\left(z_{1}, z_{2}\right)\right) *\left(\left(y_{1}, y_{2}\right) *\left(z_{1}, z_{2}\right)\right)\right) \vee(\mu \times v)\left(y_{1}, y_{2}\right) \\
= & (\mu \times v)\left(\left(x_{1} * z_{1}, x_{2} * z_{2}\right) *\left(y_{1} * z_{1}, y_{2} * z_{2}\right)\right) \vee(\mu \times v)\left(y_{1}, y_{2}\right) \\
= & (\mu \times v)\left(\left(x_{1} * z_{1}\right) *\left(y_{1} * z_{1}\right),\left(x_{2} * z_{2}\right) *\left(y_{2} * z_{2}\right)\right) \vee(\mu \times v)\left(y_{1}, y_{2}\right) \\
= & \left(\mu\left(\left(x_{1} * z_{1}\right) *\left(y_{1} * z_{1}\right)\right) \vee v\left(\left(x_{2} * z_{2}\right) *\left(y_{2} * z_{2}\right)\right)\right) \vee\left(\mu\left(y_{1}\right) \vee v\left(y_{2}\right)\right) \\
= & \left(\mu\left(\left(x_{1} * z_{1}\right) *\left(y_{1} * z_{1}\right)\right) \vee \mu\left(y_{1}\right)\right) \vee\left(v\left(\left(x_{2} * z_{2}\right) *\left(y_{2} * x_{2}\right)\right) \vee v\left(y_{2}\right)\right) \\
\leq & \mu\left(x_{1}\right) \vee v\left(x_{2}\right) \\
= & (\mu \times v)\left(x_{1}, x_{2}\right) .
\end{aligned}
$$

This completes the proof.

THEOREM 3.9. Let $\mu$ and $v$ be fuzzy subsets of a BCI-algebra $X$ such that $\mu \times v$ is a DF $p$-ideal of $X \times X$. Then

(i) either $\mu(x) \geq \mu(0)$ or $v(x) \geq v(0)$ for all $x \in X$;

(ii) if $\mu(x) \geq \mu(0)$ for all $x \in X$, then either $\mu(x) \geq v(0)$ or $v(x) \geq v(0)$;

(iii) if $v(x) \geq v(0)$ for all $x \in X$, then either $\mu(x) \geq \mu(0)$ or $v(x) \geq \mu(0)$;

(iv) either $\mu$ or $v$ is a DF $p$-ideal of $X$.

Proof. (i) Suppose that $\mu(x)<\mu(0)$ and $v(y)<v(0)$ for some $x, y \in X$. Then

$$
(\mu \times v)(x, y)=\mu(x) \vee v(y)<\mu(0) \vee v(0)=(\mu \times v)(0,0) .
$$

This is a contradiction and we obtain (i).

(ii) Assume that there exist $x, y \in X$ such that $\mu(x)<v(0)$ and $v(y)<v(0)$. Then $(\mu \times v)(0,0)=\mu(0) \vee v(0)=v(0)$. It follows that $(\mu \times v)(x, y)=\mu(x) \vee v(y)<v(0)=$ $(\mu \times v)(0,0)$, which is a contradiction. Hence (ii) holds.

(iii) Its proof follows by a similar method to (ii).

(iv) Since by (i) either $\mu(x) \geq \mu(0)$ or $v(x) \geq v(0)$ for all $x \in X$; without loss of generality, we may assume that $v(x) \geq v(0)$ for all $x \in X$. From (iii) it follows that either $\mu(x) \geq \mu(0)$ or $v(x) \geq \mu(0)$. If $v(x) \geq \mu(0)$ for any $x \in X$, then $(\mu \times v)(0, x)=$ $\mu(0) \vee v(x)=v(x)$. Let $\left(x_{1}, x_{2}\right),\left(y_{1}, y_{2}\right),\left(z_{1}, z_{2}\right) \in X \times X$, since $\mu \times v$ is a DF $p$-ideal of $X \times X$. We have $(\mu \times v)\left(x_{1}, x_{2}\right) \leq(\mu \times v)\left(\left(\left(x_{1}, x_{2}\right) *\left(z_{1}, z_{2}\right)\right) *\left(\left(y_{1}, y_{2}\right) *\left(z_{1}, z_{2}\right)\right)\right) \vee$ $(\mu \times v)\left(y_{1}, y_{2}\right)=(\mu \times v)\left(\left(x_{1} * z_{1}\right) *\left(y_{1}, z_{1}\right),\left(x_{2} * z_{2}\right) *\left(y_{2} * x_{2}\right)\right) \vee(\mu \times v)\left(y_{1}, y_{2}\right)$. If we take $x_{1}=y_{1}=z_{1}=0$, then $v\left(x_{2}\right)=(\mu \times v)\left(0, x_{2}\right) \leq(\mu \times v)\left(0,\left(x_{2} * z_{2}\right) *\left(y_{2} *\right.\right.$ $\left.\left.z_{2}\right)\right) \vee(\mu \times v)\left(0, y_{2}\right)=\left(\mu(0) \vee v\left(\left(x_{2} * z_{2}\right) *\left(y_{2} * z_{2}\right)\right)\right) \vee\left(\mu(0) \vee v\left(y_{2}\right)\right)=v\left(\left(x_{2} *\right.\right.$ $\left.\left.z_{2}\right) *\left(y_{2} * z_{2}\right)\right) \vee v\left(y_{2}\right)$. This proves that $v$ is a DF $p$-ideal of $X$. Now we consider the case $\mu(x) \geq \mu(0)$ for all $x \in X$, suppose that $v(y)<\mu(0)$ for some $y \in X$. Then $v(0) \leq v(y)<\mu(0)$, since $\mu(x) \geq \mu(0)$. It follows that $\mu(x)>v(0)$ for any $x \in X$. Hence $(\mu \times v)(x, 0)=\mu(x) \vee v(0)=\mu(x)$. Taking $x_{2}=y_{2}=x_{2}=0$, then $\mu\left(x_{1}\right)=$ 
$(\mu \times v)\left(x_{1}, 0\right) \leq(\mu \times v)\left(\left(x_{1} * z_{1}\right) *\left(y_{1} * z_{1}\right), 0\right) \vee(\mu \times v)\left(y_{1}, 0\right)=\mu\left(\left(x_{1} * z_{1}\right) *\left(y_{1} *\right.\right.$ $\left.\left.z_{1}\right)\right) \vee \mu\left(y_{1}\right)$, which proves that $\mu$ is a DF $p$-ideal of $X$. Hence either $\mu$ or $v$ is a DF $p$-ideal of $X$.

THEOREM 3.10. Let $v$ be a fuzzy subset of a BCI-algebra $X$ and let $\mu_{v}$ be the smallest doubt fuzzy relation on $X$. Then $v$ is a DF $p$-ideal of $X$ if and only if $\mu_{v}$ is a DF $p$-ideal of $X \times X$.

Proof. Assume that $v$ is a DF $p$-ideal of $X$, we note that $\mu_{v}(0,0)=v(0) \vee v(0) \leq$ $v(x) \vee v(y)$ for all $(x, y) \in X \times X$

$$
\begin{aligned}
\mu_{v}\left(x_{1}, x_{2}\right) & =v\left(x_{1}\right) \vee v\left(x_{2}\right) \\
& \leq\left(v\left(\left(x_{1} * z_{1}\right) *\left(y_{1} * z_{1}\right)\right) \vee v\left(y_{1}\right)\right) \vee\left(v\left(\left(x_{2} * z_{2}\right) *\left(y_{1} * z_{2}\right)\right) \vee v\left(y_{2}\right)\right) \\
& =\left(v\left(\left(x_{1} * z_{1}\right) *\left(y_{1} * z_{1}\right)\right) \vee v\left(\left(x_{2} * z_{2}\right) *\left(y_{2} * z_{2}\right)\right)\right) \vee\left(v\left(y_{2}\right) \vee v\left(y_{2}\right)\right) \\
& =\mu_{v}\left(\left(x_{1} * z_{1}\right) *\left(y_{1} * z_{1}\right),\left(x_{2} * z_{2}\right) *\left(y_{2} * z_{2}\right)\right) \vee \mu_{v}\left(y_{1}, y_{2}\right) \\
& =\mu_{v}\left(\left(x_{1} * z_{1}, x_{2} * z_{2}\right) *\left(y_{1} * z_{1}, y_{2} * x_{2}\right)\right) \vee \mu_{v}\left(y_{1}, y_{2}\right) \\
& =\mu_{v}\left(\left(\left(x_{1}, x_{2}\right) *\left(z_{1}, z_{2}\right)\right) *\left(\left(y_{1}, y_{2}\right) *\left(z_{1}, z_{2}\right)\right)\right) \vee \mu_{v}\left(y_{1}, y_{2}\right),
\end{aligned}
$$

for all $\left(x_{1}, x_{2}\right),\left(y_{1}, y_{2}\right),\left(z_{1}, z_{2}\right) \in X \times X$. Hence $\mu_{v}$ is a DF p-ideal of $X \times X$.

Conversely, suppose that $\mu_{v}$ is a DF $p$-ideal of $X \times X$. Then for all $\left(x_{1}, x_{2}\right) \in X \times X$, $v(0) \vee v(0)=\mu_{v}(0,0) \leq \mu_{v}(x, x)=v(x) \vee v(x)$. It follows that $v(0) \leq v(x)$ for all $x \in X$. Now let $\left(x_{1}, x_{2}\right),\left(y_{1}, y_{2}\right),\left(z_{1}, z_{2}\right) \in X \times X$. Then

$$
\begin{aligned}
v\left(x_{1}\right) \vee v\left(x_{2}\right) & =\mu_{v}\left(x_{1}, x_{2}\right) \\
& \leq \mu_{v}\left(\left(\left(x_{1}, x_{2}\right) *\left(z_{1}, z_{2}\right)\right) *\left(\left(y_{1}, y_{2}\right) *\left(z_{1}, z_{2}\right)\right)\right) \vee \mu_{v}\left(y_{1}, y_{2}\right) \\
& =\mu_{v}\left(\left(x_{1} * x_{2}, x_{2} * z_{2}\right) *\left(y_{1} * z_{1}, y_{2} * z_{2}\right)\right) \vee \mu_{v}\left(y_{1}, y_{2}\right) \\
& =\mu_{v}\left(\left(x_{1}, z_{1}\right) *\left(y_{1} * z_{1}\right),\left(x_{2} * z_{2}\right) *\left(y_{2} * z_{2}\right)\right) \vee \mu_{v}\left(y_{1}, y_{2}\right) \\
& =\left(v\left(\left(x_{1} * z_{1}\right) *\left(y_{1} * z_{1}\right)\right) \vee v\left(y_{1}\right)\right) \vee\left(v\left(\left(x_{2} * z_{2}\right) *\left(y_{2} * x_{2}\right)\right) \vee v\left(y_{2}\right)\right) .
\end{aligned}
$$

In particular, if we take $x_{2}=y_{2}=z_{2}=0$ (resp., $\left.x_{1}=y_{1}=z_{1}=0\right)$ then $v\left(x_{1}\right) \leq$ $v\left(\left(x_{1} * z_{1}\right) *\left(y_{1} * z_{1}\right)\right) \vee v\left(y_{1}\right)$ (resp., $\left.v\left(x_{2}\right) \leq\left(\left(x_{2} * z_{2}\right) *\left(y_{2} * z_{2}\right)\right) \vee v\left(y_{2}\right)\right)$. This completes the proof.

\section{REFERENCES}

[1] P. Bhattacharya and N. P. Mukherjee, Fuzzy relations and fuzzy groups, Inform. Sci. 36 (1985), no. 3, 267-282.

[2] C. L. Chang, Fuzzy topological spaces, J. Math. Anal. Appl. 24 (1968), 182-190.

[3] F. Y. Huang, Another definition of fuzzy BCI-algebras, Selected Papers on BCK/BCI-Algebras (China), vol. 1, 1992, pp. 91-92.

[4] Y. B. Jun, Doubt fuzzy BCK/BCI-algebras, Soochow J. Math. 20 (1994), no. 3, 351-358.

[5] Y. B. Jun and J. Meng, Fuzzy p-ideals in BCI-algebras, Math. Japon. 40 (1994), no. 2, 271282.

[6] A. Rosenfeld, Fuzzy groups, J. Math. Anal. Appl. 35 (1971), 512-517. 
[7] O. Xi, Fuzzy BCK-algebra, Math. Japon. 36 (1991), no. 5, 935-942.

Zhan Jianming: Department of Mathematics, Hubei Institute for Nationalities, Enshi City, Hubei Province, 445000, China

E-mail address: zhanjianming@hotmai 1 .com

Tan Zhisong: Department of Mathematics, Hubei Institute Enshi, Hubei Province, 44500, CHINA 


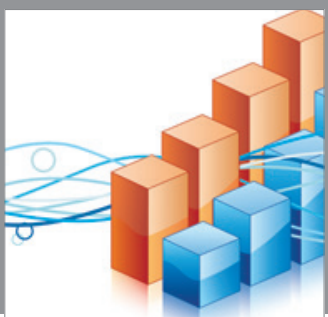

Advances in

Operations Research

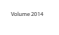

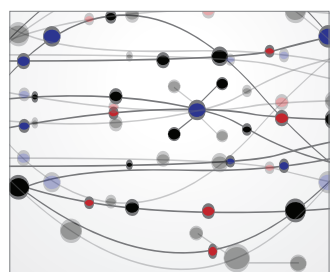

\section{The Scientific} World Journal
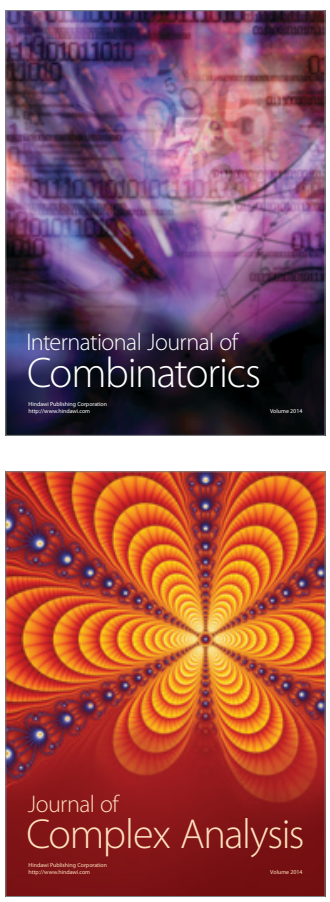

International Journal of

Mathematics and

Mathematical

Sciences
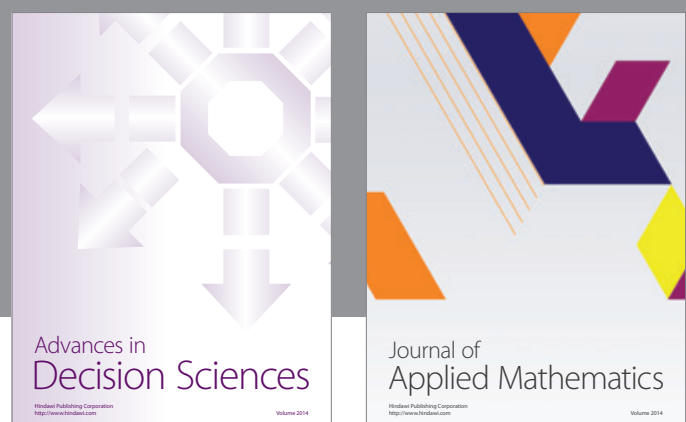

Journal of

Applied Mathematics
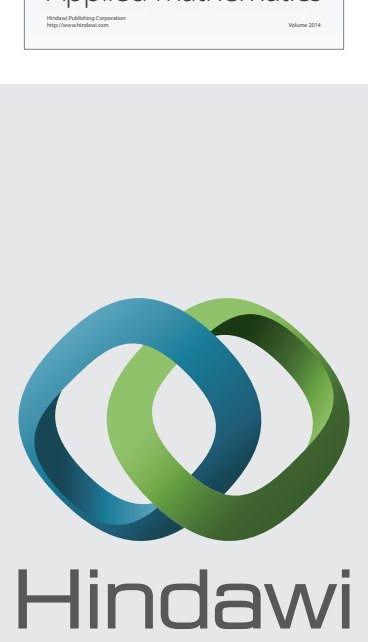

Submit your manuscripts at http://www.hindawi.com
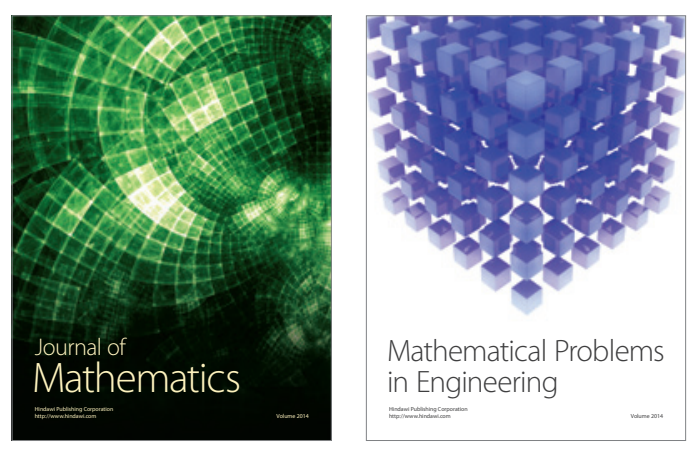

Mathematical Problems in Engineering
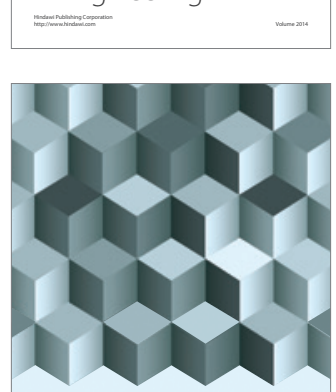

Journal of

Function Spaces
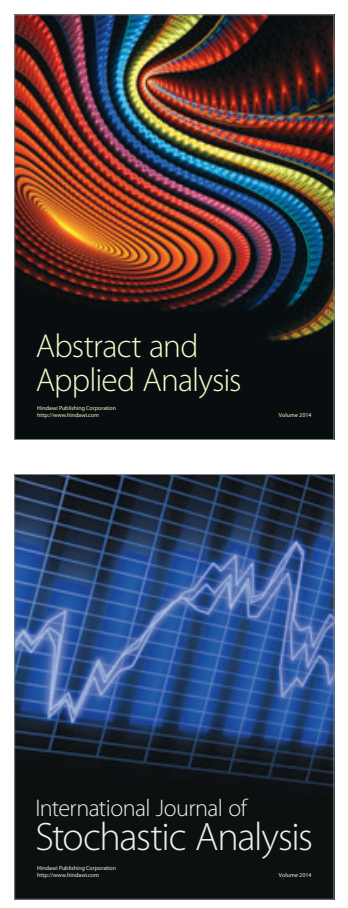

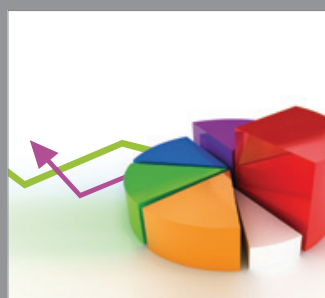

ournal of

Probability and Statistics

Promensencen
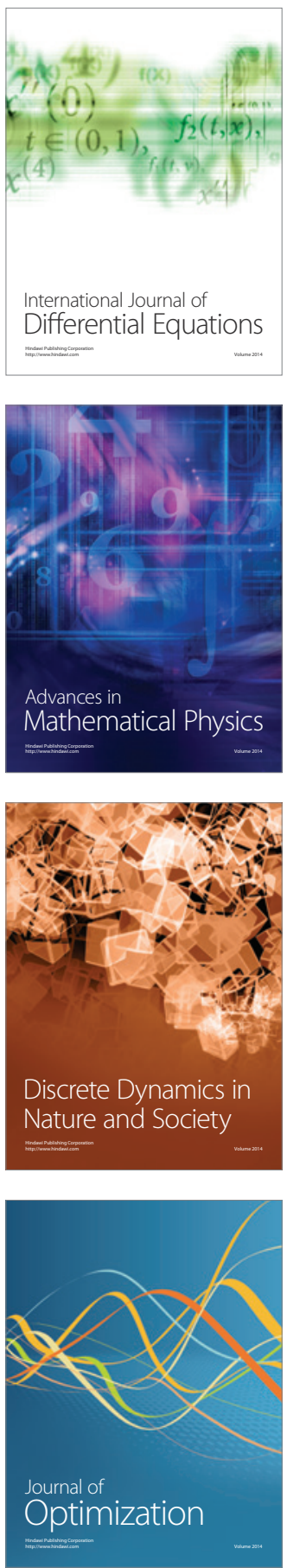